\title{
A New Approach to Difficult Fischer Synthesis: the Use of Zinc Chloride Catalyst in Triethylene Glycol under Controlled Microwave Irradiation.
}

\author{
Teodozja M. Lipińska, ${ }^{*}$ Stefan J. Czarnocki \\ Institute of Chemisty, University of Podlasie, ul. 3 Maja 54, 08-110 Siedlce, Poland \\ tlip@ap.siedlce.pl
}

\section{Experimental Section}

\begin{abstract}
General
Starting materials 5a-d were prepared from 3methylthio-1,24-triazine ${ }^{1}$ via three steps procedure described previously. ${ }^{2} \quad$ Microwave-assisted syntheses were performed with the Synthewave 402 microwave reactor (Prolabo, $300 \mathrm{~W}$, open rotating system of a reaction vessel, infrared temperature detector) interfaced with the software for feedback temperature monitoring. Commercial phenylhydrazine and $p$-methoxyphenylhydrazine hydrochloride was used. Anhydrous zinc chloride was obtained ${ }^{3}$ by melting and heating at temperature above $300{ }^{\circ} \mathrm{C}$ and next cooled in dessicator over $\mathrm{P}_{2} \mathrm{O}_{5} .{ }^{3}$ Triethylene glycol was dried, distillied under vacum and stored over molecular sieves under argon. Other organic solvents: ethanol, hexane, dichloromethane; were routinally dried $^{3}$ or/and distillied prior to use and stored over molecular sieves. The course of reactions was monitored by thin-layer chromatography (TLC), which was carried out on $0.25 \mathrm{~mm}$ Merck silica gel $\left(60 \mathrm{~F}_{254}\right)$ plates on aluminium. Column

chromatography was performed on Merck silica gel 60 (230-400 mesh). The column output was

monitored with TLC. Melting points were determined on Boetius microscopic plate and are uncorected. All
\end{abstract}

new compouds were determined to be $>97 \%$ pure by ${ }^{1} \mathrm{H}$ NMR and GC/MS analyses. ${ }^{1} \mathrm{H}$ and ${ }^{13} \mathrm{C}$ NMR spectra were recorded with Varian Gemini (200 $\mathrm{MHz})$ and Mercury $400 \mathrm{BB}(400 \mathrm{MHz})$ spectrometers. GC MS analyses were performed with a Shimadzu MS-QP555 mass chromatograph interfaced with selective mass detector (column: Zebron ZB-5, $30 \mathrm{M}$ x $0.25 \mathrm{~mm}$, IDx $0.10 \mu \mathrm{m})$. IR spectra ( $\mathrm{KBr}$ pellets) were recorded on FT-IR Magna 760 (Nicolet) apparatus. High resolution EI mass spectra were obtained with an AMD 604 (Inectra, $\mathrm{GmbH}$, Germany) spectrometer.

\section{Characterization data for indoles 3a-d}

2-(1-Methylthio-6,7-dihydro-5H-[2]pyrindin-3yl)-1 $H$-indole (3a):

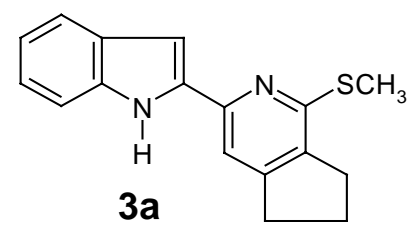


Colorless crystals with m.p $102-103{ }^{0} \mathrm{C}$ (from dichloroethane/hexane); $R_{\mathrm{f}}=0.84$ (dichloromethane); GC/MS: $t_{\mathrm{R}}=16.8 \mathrm{~min} ; \mathrm{MS}(\mathrm{EI}): \mathrm{m} / z$ (\%) 280 (51, $\mathrm{M}^{+}$), 265 (12), 247 (100), 238 (8), 232 (10), 218 (3), 204 (10), 140(7, M $\left.{ }^{2+}\right), 132$ (3), 123 (8), 116 (10), 102 (10); IR (KBr): 3447, 3429, 3049, 2942, 2837, $1589,1555,1421,1342,1301,1216,1127 ;{ }^{1} \mathrm{H}$ NMR (400 MHz, $\mathrm{CDCl}_{3}$ ); $\delta 2.19$ (quintet, $J=7.5 \mathrm{~Hz}$, $\left.2 \mathrm{H}, H_{2} \mathrm{C}-6\right), 2.55$ (s, 3H, $\left.H_{3} \mathrm{CS}\right), 2.74$ (t, J=7.5 Hz, $\left.2 \mathrm{H}, H_{2} \mathrm{C}-7\right), 2.89$ (t, $\left.J=7.5 \mathrm{~Hz}, 2 \mathrm{H}, H_{2} \mathrm{C}-5\right), 6.96(\mathrm{dd}$, $\left.J=2.0 \mathrm{~Hz}, \mathrm{~J}=0.9 \mathrm{~Hz}, 1 \mathrm{H}, H \mathrm{C}-3_{\text {ind. }}\right), 7.10-7.28(\mathrm{~m}, 2 \mathrm{H}$, $H \mathrm{C}-5-H \mathrm{C}-6_{\text {ind }}$ ), 7.42 (s, 1H, HC-4), 7.46 (dd, $J=7.9$ $\left.\mathrm{Hz}, J=1.1 \mathrm{~Hz}, 1 \mathrm{H}, H \mathrm{C}-7_{\text {ind }}\right), 7.66(\mathrm{dd}, J=7.0 \mathrm{~Hz}$, $\left.J=1.3 \mathrm{~Hz}, 1 \mathrm{H}, H \mathrm{C}-4_{\text {ind }}\right), 9.39$ (br s, $\left.1 \mathrm{H}, H \mathrm{~N}_{\text {ind }}\right) ;{ }^{13} \mathrm{C}$ NMR $\left(100 \mathrm{MHz}, \mathrm{CDCl}_{3}\right): \delta 12.7\left(\mathrm{SCH}_{3}\right), 24.2$ $\left(\mathrm{CH}_{2}\right), 30.0\left(\mathrm{CH}_{2}\right), 32.8\left(\mathrm{CH}_{2}\right), 99.5(\mathrm{CH}), 111.1$ $(\mathrm{CH}), 111.8(\mathrm{CH}), 120.0(\mathrm{CH}), 120.9(\mathrm{CH}), 122.7$ $(C \mathrm{H}), 129.2(C), 135.8(C), 136.0(C), 137.4(C)$, $148.0(C), 153.5(C), 153.8(C)$; HRMS (EI, $\left.\mathrm{M}^{+}\right)$: Found 280.1035, calcd for $\mathrm{C}_{17} \mathrm{H}_{16} \mathrm{~N}_{2} \mathrm{~S} 280.1034$.

\section{3-(Indol-2-yl)-1-methylthio-5,6,7,8-tetrahydroiso- quinoline (3b):}

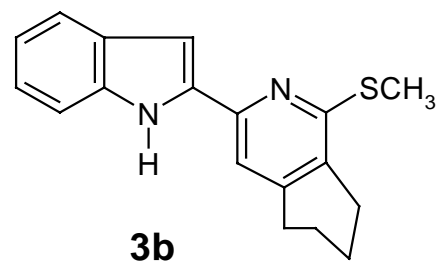

Colorless crystals with m.p $97-98{ }^{0} \mathrm{C}$ (from dichloroethane/hexane), described in our previous paper, ${ }^{4}$ m.p $106-107{ }^{0} \mathrm{C}$ (after sublimation). The new data: $R_{\mathrm{f}}=0.85$ (dichloromethane); GC/MS: $t_{\mathrm{R}}=17.6$ min; MS(EI): $m / z$ (\%) $294\left(64, \mathrm{M}^{+}\right), 279$ (41), 261 (100), 245 (9), 216 (6), 204 (4), $147\left(7, \mathrm{M}^{2+}\right), 139$ (5), 123 5), 117 (4), 116 (4), 108 (5); IR (KBr); $3444,3055,2953,2839,1586,1552,1420,1342$, 1300, 1218, 1125; ${ }^{1} \mathrm{H}$ NMR (400 MHZ, $\left.\mathrm{CDCl}_{3}\right): \delta$ 1.73-1.96 (m, 4H, $\left.H_{2} \mathrm{C}-6-H_{2} \mathrm{C}-7\right), 2.62(\mathrm{t}, J=6.0 \mathrm{~Hz}$, $\left.2 \mathrm{H}, \mathrm{H}_{2} \mathrm{C}-8\right), 2.71\left(\mathrm{~s}, 3 \mathrm{H}, H_{3} \mathrm{CS}\right), 2.77 \quad(\mathrm{t}, J=6.0 \mathrm{~Hz}$, $\left.2 \mathrm{H}, H_{2} \mathrm{C}-5\right), 6.94$ (dd, $J=2.0 \mathrm{~Hz}, J=0.9 \mathrm{~Hz}, 1 \mathrm{H}, H \mathrm{C}-$ $\left.3_{\text {ind }}\right), 7.08-7.20\left(\mathrm{~m}, 2 \mathrm{H}, H \mathrm{C}-5-H \mathrm{C}-6_{\text {ind }}\right), 7.24(\mathrm{~s}, 1 \mathrm{H}$, $H \mathrm{C}-4), 7.46$ (dd, $J=7.9 \mathrm{~Hz}, J=1.0 \mathrm{~Hz}, 1 \mathrm{H}, H \mathrm{C}-7_{\text {ind }}$ ), 7.66 (dd $J=7.5 \mathrm{~Hz}, J=1.3 \mathrm{~Hz}, 1 \mathrm{H}, H \mathrm{C}-4_{\text {ind }}$ ), 9.33 (br $\left.\mathrm{s}, 1 \mathrm{H}, H \mathrm{~N}_{\text {ind }}\right) ;{ }^{13} \mathrm{C} \mathrm{NMR}\left(100 \mathrm{MHz}, \mathrm{CDCl}_{3}\right): \delta 13.2$ $\left(\mathrm{SCH}_{3}\right), 22.1\left(\mathrm{CH}_{2}\right), 22.6\left(\mathrm{CH}_{2}\right), 25.00\left(\mathrm{CH}_{2}\right), 29.3$ $\left(\mathrm{CH}_{2}\right), 99.3(\mathrm{CH}), 111.1(\mathrm{CH}), 115.8(\mathrm{CH}), 120.0$ $(C \mathrm{H}), 120.9(\mathrm{CH}), 122.6(\mathrm{CH}), 128.9(C), 129.3$ (C), $136.0(C), 137.2(C), 145.7(C), 146.1(C)$, $157.7(C)$.
3-(Indol-2-yl)-1-methylthio-6,7,8,9-tetrahydro$5 H$-cyclohepta $[c]$ pyridine (3c):

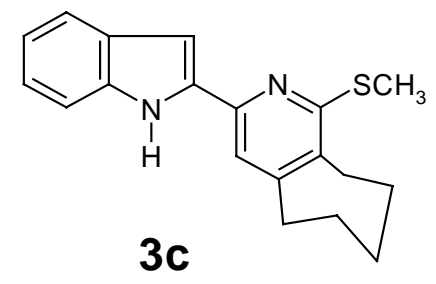

Colorless crystals with m.p $114-115{ }^{0} \mathrm{C}$ (from dichloroethane/hexane); $R_{\mathrm{f}}=0.86$ (dichloromethane); GC/MS: $t_{\mathrm{R}}=17.9 \mathrm{~min} ; \mathrm{MS}(\mathrm{EI}): \mathrm{m} / z(\%) 308(68$, $\left.\mathrm{M}^{+}\right), 293$ (44), 279 (7), 275 (100), 265 (15), 259 (6), 247 (14), 231 (7) 217 (5), 204 (5), $154\left(7, \mathrm{M}^{2+}\right), 139$ (4), 132 (8), 122 (8), 117 (8), 116 (9), 102 (7); IR (KBr); 3467, 3417, 3056, 2921, 2846, 1589, 1545, $1439,1412,1341,1307,1226,1192 ;{ }^{1} \mathrm{H}$ NMR $(400$ $\left.\mathrm{MHZ}, \mathrm{CDCl}_{3}\right): \delta 1.65-1.81\left(\mathrm{~m}, 2 \mathrm{H}, \mathrm{H}_{2} \mathrm{C}-7\right), 1.82-$ $1.98\left(\mathrm{~m}, 4 \mathrm{H},\left(\mathrm{H}_{2} \mathrm{C}-6\right.\right.$ and $\left.\mathrm{H}_{2} \mathrm{C}-8\right), 2.70(\mathrm{~s}, 3 \mathrm{H}$, $H_{3} \mathrm{CS}$ ), 2.81 (t, $J=5.4 \mathrm{~Hz}, 2 \mathrm{H}, \mathrm{H}_{2} \mathrm{C}-9$ ), 2.91 (t, $J=5.4$ $\left.\mathrm{Hz}, 2 \mathrm{H}, \mathrm{H}_{2} \mathrm{C}-5\right), 6.97(\mathrm{dd}, J=2.0 \mathrm{~Hz}, J=0.9 \mathrm{~Hz}, 1 \mathrm{H}$, $\left.H \mathrm{C}-3_{\text {ind }}\right), 7.14-7.28$ (m, $\left.2 \mathrm{H}, H \mathrm{C}-5-H \mathrm{C}-6_{\text {ind }}\right), 7.30$ (s, $1 \mathrm{H}, H \mathrm{C}-4), 7.46$ (dd, $J=7.8 \mathrm{~Hz}, J=0.9 \mathrm{~Hz}, 1 \mathrm{H}, H \mathrm{C}-$ 7 ind $), 7.67$ (dd $J=7.6 \mathrm{~Hz}, J=1.1 \mathrm{~Hz}, 1 \mathrm{H}, H \mathrm{C}-4_{\text {ind }}$ ), 9.33 (br s, $\left.1 \mathrm{H}, H \mathrm{~N}_{\text {ind }}\right) ;{ }^{13} \mathrm{C} \mathrm{NMR}\left(100 \mathrm{MHz}, \mathrm{CDCl}_{3}\right)$ : $\delta 14.1\left(\mathrm{SCH}_{3}\right), 26.6\left(\mathrm{CH}_{2}\right), 27.2\left(\mathrm{CH}_{2}\right), 29.8\left(\mathrm{CH}_{2}\right)$, $32.4\left(\mathrm{CH}_{2}\right), 36.00\left(\mathrm{CH}_{2}\right), 99.7(\mathrm{CH}), 111.1(\mathrm{CH})$, $116.3(\mathrm{CH}), 120.0(\mathrm{CH}), 121.00(\mathrm{CH}), 122.7(\mathrm{CH})$, $129.2(C), 134.5(C), 136.0(C), 137.1(C), 147.00$ $(C), 152.2(C), 156.1(C)$; HRMS (EI, $\left.\mathrm{M}^{+}\right)$: Found 308.1350, calcd for $\mathrm{C}_{19} \mathrm{H}_{20} \mathrm{~N}_{2} \mathrm{~S} 308.1347$.

\section{3-(Indol-2-yl)-1-methylthio-5,6,7,8,9,10-hexa- hydrocycloocta $[c]$ pyridine (3d):}

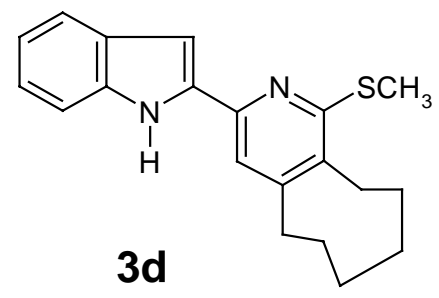

Colorless crystals with m.p 144-145 ${ }^{0} \mathrm{C}$ (from dichloroethane/hexane); $R_{\mathrm{f}}=0.87$ (dichloromethane); GC/MS: $t_{\mathrm{R}}=18.6 \mathrm{~min} ; \mathrm{MS}(\mathrm{EI}): \mathrm{m} / \mathrm{z}(\%) 322(100$, $\mathrm{M}^{+}$), 307 (86), 293 (14), 289 (67), 280 (21), 275 (24), 265 (5), 261 (7), 247 (5), 245 (4), 232 (3) 219 (3), 205 (4), 192 (3), $161\left(5, \mathrm{M}^{2+}\right), 140$ (6), 130 (3), 116 (3), 102 (3); IR (KBr); 3467, 3417, 3056, 2921, 2846, 1589, 1545, 1439, 1412, 1341, 1307, 1226, 1192; ${ }^{1} \mathrm{H}$ NMR (400 MHZ, $\left.\mathrm{CDCl}_{3}\right): \delta 1.34-1.48(\mathrm{~m}$, $\left.4 \mathrm{H}, \mathrm{H}_{2} \mathrm{C}-7-\mathrm{H}_{2} \mathrm{C}-8\right), 1.72-1.82\left(\mathrm{~m}, 4 \mathrm{H},\left(\mathrm{H}_{2} \mathrm{C}-6\right.\right.$ and $H_{2} \mathrm{C}-9$ ), 2.70 (s, 3H, $H_{3} \mathrm{CS}$ ), 2.78 (t, $J=6.2 \mathrm{~Hz}, 2 \mathrm{H}$, $\mathrm{H}_{2} \mathrm{C}-10$ ), 2.89 (t, J=6.2 Hz, 2H, $H_{2} \mathrm{C}-5$ ), 6.97 (dd, 
$J=2.0 \mathrm{~Hz}, J=0.9 \mathrm{~Hz}, 1 \mathrm{H}, H \mathrm{C}-3_{\text {ind }}$ ), 7.11 (ddd, $J=7.8$ $\mathrm{Hz}, J=7.2 \mathrm{~Hz}, J=0.9 \mathrm{~Hz}, 1 \mathrm{H}, H \mathrm{C}-5_{\text {ind }}$ ), 7.21 (ddd, $\left.J=8.0 \mathrm{~Hz}, \mathrm{~J}=7.2 \mathrm{~Hz}, J=1.0 \mathrm{~Hz}, 1 \mathrm{H}, H \mathrm{C}-6_{\text {ind }}\right), 7.31$ (s, 1H, HC-4), $7.45(\mathrm{dd}, J=8.0 \mathrm{~Hz}, J=0.9 \mathrm{~Hz}, 1 \mathrm{H}$, $\left.H C-7_{\text {ind }}\right), 7.64$ (dd $\left.J=7.8 \mathrm{~Hz}, J=1.0 \mathrm{~Hz}, 1 \mathrm{H}, H \mathrm{C}-4_{\text {ind }}\right)$, 9.36 (br s, $\left.1 \mathrm{H}, H \mathrm{~N}_{\text {ind }}\right) ;{ }^{13} \mathrm{C} \mathrm{NMR}\left(100 \mathrm{MHz}, \mathrm{CDCl}_{3}\right)$ : $\delta 13.8\left(\mathrm{SCH}_{3}\right), 26.0\left(\mathrm{CH}_{2}\right), 26.1\left(\mathrm{CH}_{2}\right), 26.7\left(\mathrm{CH}_{2}\right)$, $28.4\left(\mathrm{CH}_{2}\right), 31.7\left(\mathrm{CH}_{2}\right), 32.6\left(\mathrm{CH}_{2}\right), 99.7(\mathrm{CH})$, $111.2(\mathrm{CH}), 116.4(\mathrm{CH}), 120.0(\mathrm{CH}), 121.00(\mathrm{CH})$, $122.8(\mathrm{CH}), 129.2(C), 132.3(C), 136.1(C), 136.9$ (C), $147.1(C), 150.7(C), 156.8(C)$; HRMS (EI, $\left.\mathrm{M}^{+}\right)$: Found 322.1500, calcd for $\mathrm{C}_{20} \mathrm{H}_{22} \mathrm{~N}_{2} \mathrm{~S}$ 322.1504 .

\section{Characterization data for 6-methoxyindoles 4a-d}

\section{5-Methoxy-2-(1-methylthio-6,7-dihydro-5H-} [2]pyrindin-3-yl)-1H-indole (4a):

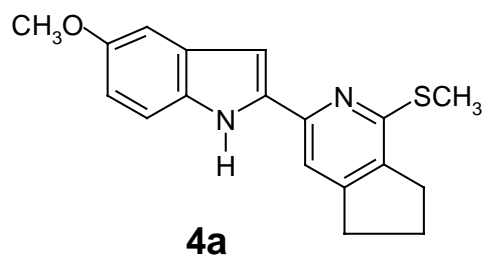

Colorless crystals with m.p 193-194 ${ }^{0} \mathrm{C}$ (from dichloroethane/hexane); $R_{\mathrm{f}}=0.51$ (dichloromethane); GC/MS: $t_{\mathrm{R}}=18.3 \mathrm{~min} ; \mathrm{MS}(\mathrm{EI}): \mathrm{m} / \mathrm{z}$ (\%) 310 (79, $\mathrm{M}^{+}$), 295 (32), 277 (100), 267 (16), 262 (5), 251 (4), 234 (4), 232 ( 4), 219 (6), 205 (3), 192 (4), 155 (6, $\mathrm{M}^{2+}$ ), 138 (10), 131 (3), 126 (4), 116 (3), 109 (6), 96 (4); IR (KBr); 3453, 2991, 2925, 2857, 1621, 1586, $1552,1448,1415,1337,1294,1232,1210,1154$; ${ }^{1} \mathrm{H}$ NMR (400 MHZ, $\mathrm{CDCl}_{3}$ ): $\delta 2.13$ (quintet, $J=7.6$ $\left.\mathrm{Hz}, 2 \mathrm{H}, H_{2} \mathrm{C}-6\right), 2.71$ (s, 3H, $\left.H_{3} \mathrm{CS}\right), 2.81$ (t, $J=7.6$ $\mathrm{Hz}, 2 \mathrm{H}, \mathrm{H}_{2} \mathrm{C}-7$ ), 2.94 (t, J=7.6 Hz, 2H, $\left.\mathrm{H}_{2} \mathrm{C}-5\right), 3.87$ (s, $\left.3 \mathrm{H}, H_{3} \mathrm{CO}\right), 6.86-6.89$ (m, $\left.1 \mathrm{H}, H \mathrm{C}-3_{\text {ind. }}\right), 6.89$ (dd, $\left.J=8.9 \mathrm{~Hz}, \mathrm{~J}=2.4 \mathrm{~Hz}, 1 \mathrm{H}, H \mathrm{C}-6_{\text {ind }}\right), 7.09$ (d, $J=2.4 \mathrm{~Hz}$, $\left.1 \mathrm{H}, H \mathrm{C}-4_{\text {ind }}\right), 7.28\left(\mathrm{~d}, J=8.9 \mathrm{~Hz}, 1 \mathrm{H}, H \mathrm{C}-7_{\text {ind }}\right), 7.38$ (s, $1 \mathrm{H}, H \mathrm{C}-4), 9.24$ (br s, $\left.1 \mathrm{H}, H \mathrm{~N}_{\text {ind }}\right) ;{ }^{13} \mathrm{C}$ NMR $(100$ $\left.\mathrm{MHz}, \mathrm{CDCl}_{3}\right): \delta 12.7\left(\mathrm{SCH}_{3}\right), 24.2\left(\mathrm{CH}_{2}\right), 30.00$ $\left(\mathrm{CH}_{2}\right), 32.8\left(\mathrm{CH}_{2}\right), \quad 55.8\left(\mathrm{CH}_{3} \mathrm{O}\right), 99.2(\mathrm{CH}), 102.2$ $(\mathrm{CH}), 111.7(\mathrm{CH}), 111.9(\mathrm{CH}), 113.2(\mathrm{CH}), 129.6$ (C), $131.3(C), 135.7(C), 138.0(C), 147.9(C)$, $153.4(C), 153.7(C), 154.3(C)$; HRMS (EI, $\left.\mathrm{M}^{+}\right)$: Found 310.1151, calcd for $\mathrm{C}_{18} \mathrm{H}_{18} \mathrm{~N}_{2} \mathrm{SO} 310.1140$.

3-(5-Methoxyindol-2-yl)-1-methylthio-5,6,7,8tetrahydroisoquinoline $(4 \mathrm{~b})$ :

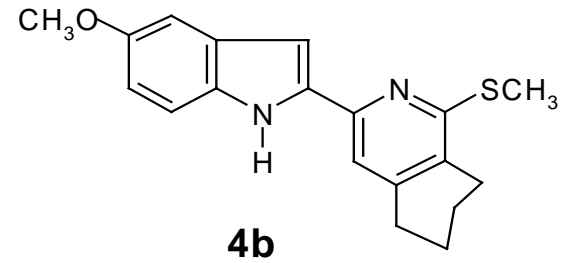

Colorless crystals with m.p $178-179{ }^{0} \mathrm{C}$ (from dichloroethane/hexane); $R_{\mathrm{f}}=0.53$ (dichloromethane); GC/MS: $t_{\mathrm{R}}=19.5 \mathrm{~min} ; \mathrm{MS}(\mathrm{EI}): \mathrm{m} / z(\%) 324(98$, $\mathrm{M}^{+}$), 309 (61), 291 (100), 281 (10), 276 (8), 265 (3), 250 (4), 233 (4), 219 (3), 207 (3), 192 (3), 162 (10, $\mathrm{M}^{2+}$ ), 154 (8), 147 (4), 133 (4), 116 (7), 102 (10), 96 (3); IR (KBr); 3487, 3060, 3001, 2926, 2856, 2831, 1622, 1583, 1549, 1487 1451, 1405, 1353, 1297, $1229,1209,1157,1120 ;{ }^{1} \mathrm{H}$ NMR $(200 \mathrm{MHz}$, $\left.\left.\mathrm{CDCl}_{3}\right) \delta 1.75-1.95(\mathrm{~m}, 4 \mathrm{H}), \mathrm{H}_{2} \mathrm{C}-6-\mathrm{H}_{2} \mathrm{C}-7\right), 2.61(\mathrm{t}$, $\left.J=5.6 \mathrm{~Hz}, 2 \mathrm{H}, H_{2} \mathrm{C}-8\right), 2.69\left(\mathrm{~s}, 3 \mathrm{H}, H_{3} \mathrm{CS}\right), 2.77 \quad(\mathrm{t}$, $\left.J=5.6 \mathrm{~Hz}, 2 \mathrm{H}, \mathrm{H}_{2} \mathrm{C}-5\right), 3.88\left(\mathrm{~s}, 3 \mathrm{H}, \mathrm{H}_{3} \mathrm{CO}\right), 6.89$ (dd, $\left.J=8.8 \mathrm{~Hz}, J=2.4 \mathrm{~Hz}, 1 \mathrm{H}, H \mathrm{C}-6_{\text {ind }}\right) 6.94$ (br s, $\left.1 \mathrm{H}, H \mathrm{C}-3_{\text {ind. }}\right), 7.09$ (d, $\left.J=2.4 \mathrm{~Hz}, 1 \mathrm{H}, H \mathrm{C}-4_{\text {ind }}\right), 7.21$ (s, $1 \mathrm{H}, H \mathrm{C}-4), 7.34$ (d, $\left.J=8.8 \mathrm{~Hz}, 1 \mathrm{H}, H \mathrm{C}-7_{\text {ind }}\right), 9.22$ (br s, $\left.1 \mathrm{H}, H \mathrm{~N}_{\text {ind }}\right) ;{ }^{13} \mathrm{C}$ NMR $\left(50 \mathrm{MHz}, \mathrm{CDCl}_{3}\right): \delta$ $13.2\left(\mathrm{SCH}_{3}\right), 22.1\left(\mathrm{CH}_{2}\right), 22.6\left(\mathrm{CH}_{2}\right), 24.9\left(\mathrm{CH}_{2}\right)$, $29.3\left(\mathrm{CH}_{2}\right), 55.8\left(\mathrm{CH}_{3} \mathrm{O}\right), 99.1(\mathrm{CH}), 102.2(\mathrm{CH})$, $111.8(\mathrm{CH}), 113.0(\mathrm{CH}), 115.6(\mathrm{CH}), 128.8(\mathrm{C})$, $129.7(C), 131.3(C), 137.8(C), 145.7(C), 146.1$ (C), $154.3(C), 157.7(C)$; HRMS EI; found 324.1303, calcd for $\mathrm{C}_{19} \mathrm{H}_{20} \mathrm{ON}_{2} \mathrm{~S} 324.1296$.

\section{3-(5-Methoxyindol-2-yl)-1-methylthio-6,7,8,9- tetrahydro-5H-cyclohepta[c]pyridine (4a):}

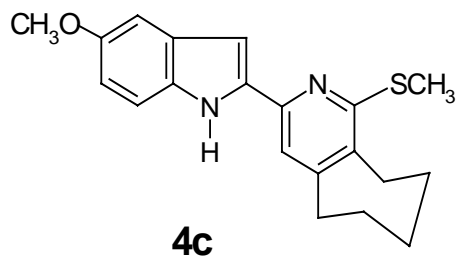

Colorless crystals with m.p 182-183 ${ }^{\circ} \mathrm{C}$ (from dichloroethane/hexane), described in our previous paper. $^{5}$ The additional data: $R_{\mathrm{f}}=0.56$ (dichloromethane); GC/MS: $t_{\mathrm{R}}=20.0 \mathrm{~min}$; $\mathrm{MS}(\mathrm{EI})$ : $\mathrm{m} / \mathrm{z}$ (\%) 338 (100, $\left.\mathrm{M}^{+}\right), 323$ (46), 305 (75), 295 (15), 290 (7), 277 (7), 262 (4), 247 (4), 234 (3), 219 (3), 205 (3), 192 (3), $169\left(9, \mathrm{M}^{2+}\right), 162$ (5), 154 (4), 147 (3), 140 (7), 126 (3), 123 (3), 116 (4), 109 (3), 102 (3).

3-(Methoxyindol-2-yl)-1-methylthio-5,6,7,8,9,10hexahydrocycloocta $[c]$ pyridine $(4 \mathrm{~d})$ : 


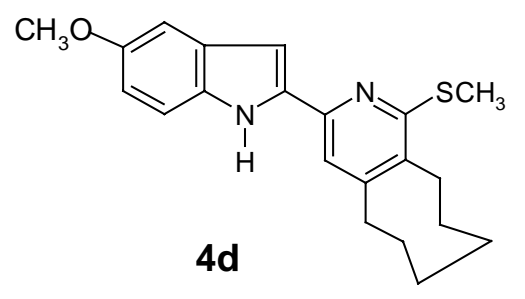

Colorless crystals with m.p 142-143 ${ }^{0} \mathrm{C}$ (from dichloroethane/hexane); $R_{\mathrm{f}}=0.58$ (dichloromethane); GC/MS: $t_{\mathrm{R}}=20.9 \mathrm{~min} ; \mathrm{MS}(\mathrm{EI}): \mathrm{m} / \mathrm{z}(\%) 352(100$, $\mathrm{M}^{+}$), 337 (29), 323 (5), 319 (17), 309 (8), 305 (7), 291 (4), 277 (3), 265 (3), 251 (3), 233 (3), 219 (3), 205 (3), 192 (3), 176 (5) 162 (3), 155 (6), 147 (4, $\mathrm{M}^{2+}$ ), 131 (3), 116 (5), 102 (4), 96 (3); IR (KBr); $3465,3058,2995,2919,2884,1625,1587,1542$, $14871449,1406,1348,1298,1233,1212,1156$, 1118; ${ }^{1} \mathrm{H}$ NMR $\left(400 \mathrm{MHz}, \mathrm{CDCl}_{3}\right) \delta 1.30-1.48(\mathrm{~m}$, $\left.4 \mathrm{H}, \mathrm{H}_{2} \mathrm{C}-7-\mathrm{H}_{2} \mathrm{C}-8\right), 1.68-1.88\left(\mathrm{~m}, 4 \mathrm{H},\left(\mathrm{H}_{2} \mathrm{C}-6\right.\right.$ and $\left.H_{2} \mathrm{C}-9\right), 2.68$ (s, 3H, $\left.H_{3} \mathrm{CS}\right), 2.76(\mathrm{t}, J=6.1 \mathrm{~Hz}, 2 \mathrm{H}$, $\left.H_{2} \mathrm{C}-10\right), 2.88$ (t, $\left.J=6.3 \mathrm{~Hz}, 2 \mathrm{H}, H_{2} \mathrm{C}-5\right), 3.87(\mathrm{~s}, 3 \mathrm{H}$, $H_{3} \mathrm{CO}$ ), 6.86-6.89 (m, $\left.1 \mathrm{H}, H \mathrm{C}-3_{\text {ind }}\right), 6.90$ (dd, $J=9.0$ $\left.\mathrm{Hz}, \mathrm{J}=2.2 \mathrm{~Hz}, 1 \mathrm{H}, H \mathrm{C}-6_{\text {ind }}\right), 7.09$ (d, $J=2.2 \mathrm{~Hz}, 1 \mathrm{H}$, $\left.H \mathrm{C}-4_{\text {ind }}\right), 7.26(\mathrm{~s}, 1 \mathrm{H}, H \mathrm{C}-4), 7.33(\mathrm{~d}, J=9.0 \mathrm{~Hz}, 1 \mathrm{H}$, $\left.H \mathrm{C}-7_{\text {ind }}\right), \quad 9.22\left(\right.$ br s, $\left.1 \mathrm{H}, H \mathrm{~N}_{\text {ind }}\right) ;{ }^{13} \mathrm{C}$ NMR $(100$ $\left.\mathrm{MHz}, \mathrm{CDCl}_{3}\right): \delta 13.7\left(\mathrm{SCH}_{3}\right), 25.9\left(\mathrm{CH}_{2}\right), 26.1$ $\left(\mathrm{CH}_{2}\right), 26.7\left(\mathrm{CH}_{2}\right), 28.4\left(\mathrm{CH}_{2}\right), 31.7\left(\mathrm{CH}_{2}\right), 32.5$ $\left(\mathrm{CH}_{2}\right), 55.7\left(\mathrm{CH}_{3} \mathrm{O}\right), 99.3(\mathrm{CH}), 102.2(\mathrm{CH}), 111.7$ $(C \mathrm{H}), 113.1(\mathrm{CH}), 116.1(\mathrm{CH}), 129.6(C), 131.3(C)$, $132.0(C), 137.5(C), 147.1(C), 150.3(C), 154.2$ (C), $156.8(C)$; HRMS EI; found 352.1607, calcd for $\mathrm{C}_{21} \mathrm{H}_{24} \mathrm{ON}_{2} \mathrm{~S} 352.1609$.

References:

1. Paudler, W. W.; Chen,T.-L. J. Heterocycl. Chem. 1970, 7, 767-771.

2. 2. Lipińska, T. Tetrahedron 2005, 61, 81488158.

3. Perrin, D. D.; Amarego, W. L. Purification of Laboratory Chemicals; Pergamon: Oxford, 1992.

4. Rykowski, A.; Lipińska, T. Synth. Commun. 1996, 26, 4409-4414.

5. Lipińska, T. Tetrahedron Lett. 2004, 45, 8831-8834 